Supporting Information

\title{
Femtosecond Laser-Induced Graphitization of Transparent Cellulose Nanofiber Films
}

\author{
Fumiya Morosawa $^{\dagger}$, Shuichiro Hayashi ${ }^{\dagger}$, Mitsuhiro Terakawa ${ }^{\dagger,+}, *$ \\ $\dagger$-School of Integrated Design Engineering, Keio University, 3-14-1 Hiyoshi, Kohoku-ku, \\ Yokohama, Kanagawa 223-8522, Japan \\ ! -Department of Electronics and Electrical Engineering, Keio University, 3-14-1 Hiyoshi, \\ Kohoku-ku, Yokohama, 223-8522, Japan \\ * Corresponding Author \\ * terakawa@elec.keio.ac.jp
}

Number of Pages : 5

Number of Figures : 4 


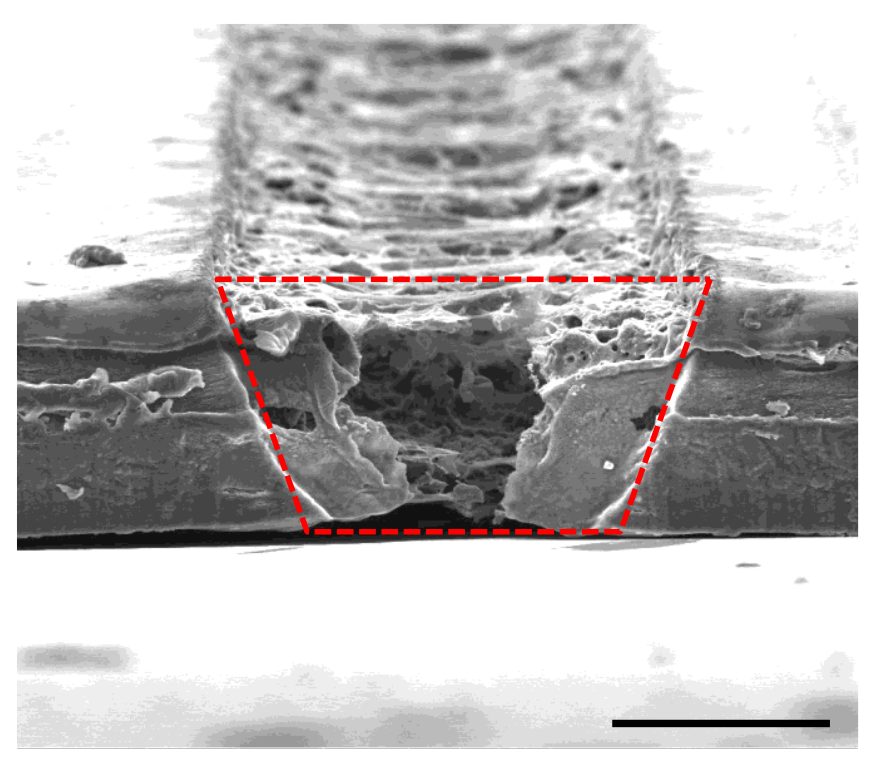

Figure S1. A typical cross-sectional SEM image of the fabricated structure. The area indicated by red line (ie. trapezoid) was used to calculate the cross-sectional area. Scale indicates $50 \mu \mathrm{m}$. 
(a)

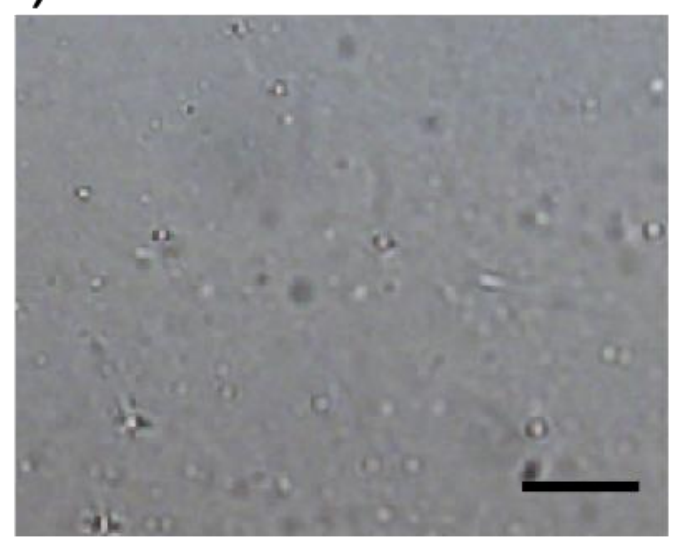

(b)

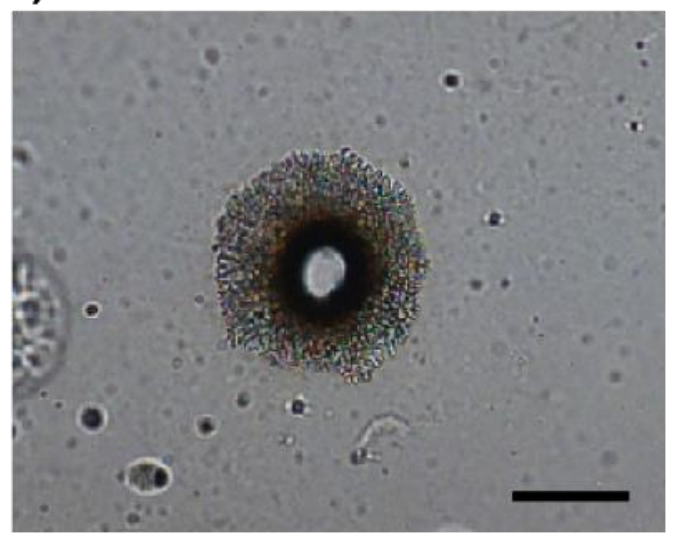

Figure S2. Optical microscope images of the CNF surface that was irradiated by a (a) defocused laser beam, and a (b) focused laser beam. The black structure (indicated in (Step 1) of Figure 1b) was fabricated only when a focused laser beam was irradiated. Scale bar indicates $100 \mu \mathrm{m}$. 


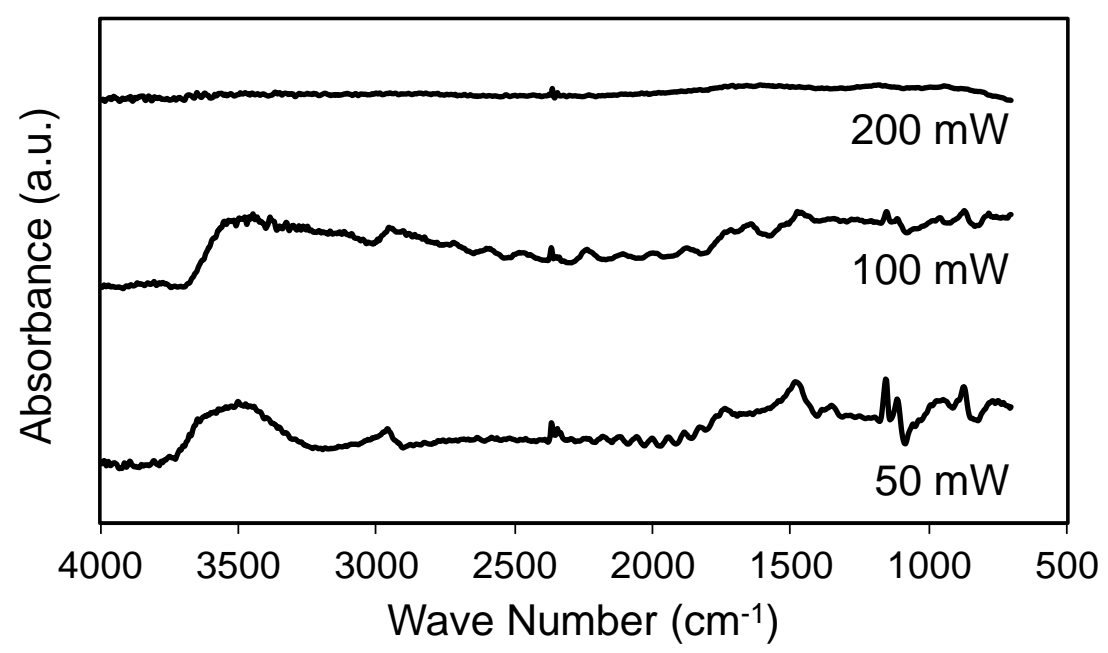

Figure S3. FT-IR spectrum obtained from the structure fabricated with a defocus distance of $100 \mu \mathrm{m}$ using laser powers of $50 \mathrm{~mW}, 100 \mathrm{~mW}$, and $200 \mathrm{~mW}$. 
(a)

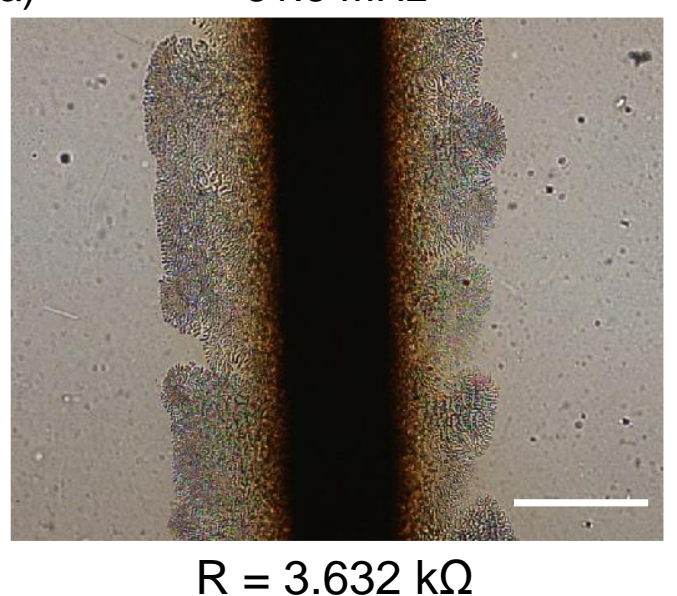

(b)

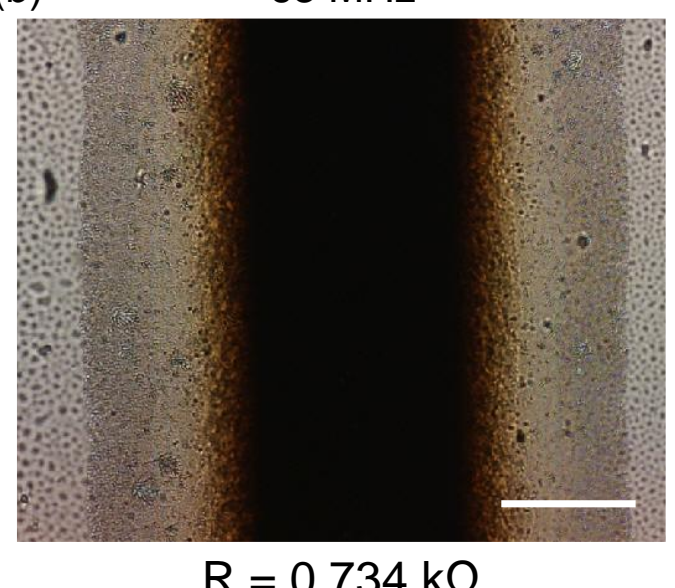

Figure S4. Optical microscope images of structures fabricated with a repetition rate of (a) 31.5 MHz and (b) $63 \mathrm{MHz}$. A laser power of $200 \mathrm{~mW}$ and a defocus distance of $100 \mu \mathrm{m}$ was used for fabrication. To keep the number of pulses constant, the scan speed was set at (a) 125 $\mu \mathrm{m} / \mathrm{s}$ and (b) $250 \mu \mathrm{m} / \mathrm{s}$. Electrical resistances measured for the structures fabricated with the respective repetition rates are indicated. Scale bar indicates $100 \mu \mathrm{m}$. 